\title{
Linear programming bounds for unitary space time codes
}

\author{
Jean Creignou Hervé Diet
}

October 25, 2018

\begin{abstract}
The linear programming method is applied to the space $\mathbb{U}_{n}(\mathbb{C})$ of unitary matrices in order to obtain bounds for codes relative to the diversity sum and the diversity product. Theoretical and numerical results improving previously known bounds are derived.
\end{abstract}

\section{Introduction}

Nowadays breakthrough of wireless communications has provided new and nice problems to the field of coding theory. Indeed strategic issues of MIMO communications has lead to consider coding in Grassmann and Stiefel manifolds, and also in unitary groups [13. Codes in unitary groups are useful in the context of non-coherent flat Rayleigh channel as shown in [ $[$. The performance of a unitary space-time code $\mathcal{V}$ is measured (see 7]) by two functions namely the diversity sum $\Sigma \mathcal{V}$ and the diversity product $\Pi \mathcal{V}$ :

$$
\begin{gathered}
\Sigma \mathcal{V}:=\frac{1}{2 \sqrt{n}} \min \{\|x-y\|: x \neq y \in \mathcal{V}\}, \\
\Pi \mathcal{V}:=\frac{1}{2} \min \left\{|\operatorname{det}(x-y)|^{\frac{1}{n}}: x \neq y \in \mathcal{V}\right\} .
\end{gathered}
$$

Here $\|A\|$ denotes the standard Euclidean norm of complex matrices: $\|A\|^{2}=$ $\operatorname{Trace}\left(A A^{*}\right)=\sum\left|A_{i, j}\right|^{2}$. A standard problem is, given a number $N$ of points in $\mathbb{U}_{n}(\mathbb{C})$, to maximize the value of $\Sigma \mathcal{V}$ or of $\Pi \mathcal{V}$. Many authors have addressed this question, narrowing gaps between bounds and explicit constructions (see [7, 8], 10], 11]). The linear programming method, which was initially developed by Philippe Delsarte in the framework of association schemes 4, and is a powerful method to deal with such questions, has not been applied to unitary codes before. Delsarte method was successfully adapted to the compact twopoint homogeneous spaces by Kabatiansky and Levenshtein [9] and recently to more general situations like the Grassmann codes [1], the permutation codes [12], the ordered codes [2, [3]. Most of the situations mentioned above fit into a 
common framework, namely a compact group $G$ acts homogeneously on the underlying space $X$, and the representation theory of $G$ constructs a certain family of orthogonal polynomials naturally attached to $X$. Standard methods of harmonic analysis show that these polynomials hold the desired positivity property that allows for Delsarte linear programming method. This general framework is recalled in Section II, and we show in Section III that unitary codes can be treated likewise, the Schur polynomials being the associated family of orthogonal polynomials. Section IV and V present the results, both numerical and analytic, obtained by the implementation of this method. It turns out that we improve all previously known bounds concerning the diversity sum and the diversity product. Moreover it is worth pointing out that the mathod can easily be extended to more complex situations, for example a diversity function involving both $\Sigma \mathcal{V}$ and $\Pi \mathcal{V}$.

\section{The linear programming method on homoge- neous spaces}

We briefly describe the linear programming method on homogeneous spaces. For more details we refer to [9, [14, Chapter 9] for a treatment of 2-point homogeneous spaces, and to [1] for the prominent case of Grassmann codes.

Let $G$ be a compact group acting transitively and continuously on a compact space $X$, and $\tau: X \times X \rightarrow Y$ such that $\tau$ characterizes the orbits of $G$ acting on $X \times X$. We mean here that, for all $x_{1}, x_{2}, x_{1}^{\prime}, x_{2}^{\prime} \in X$,

$$
\tau\left(x_{1}, x_{2}\right)=\tau\left(x_{1}^{\prime}, x_{2}^{\prime}\right) \Leftrightarrow \exists g \in G: g\left(x_{1}, x_{2}\right)=\left(x_{1}^{\prime}, x_{2}^{\prime}\right) .
$$

Let $S$ be any subset of $Y$, we call a finite subset $\mathcal{V} \subset X$ a $S$-code if for all $c_{1} \neq c_{2} \in \mathcal{V}, \tau\left(c_{1}, c_{2}\right) \in S$.

A continuous function $P: Y \rightarrow \mathbb{C}$ is said to possess the positivity property if for any finite subset $\mathcal{V} \subset X$ and any complex function $\alpha: X \rightarrow \mathbb{C}$,

$$
\sum_{x, y \in \mathcal{V}} \alpha(x) \overline{\alpha(y)} P(\tau(x, y)) \text { is real non-negative. }
$$

A canonical example is the constant function $P_{0}=1$; non-trivial examples are given by the so called zonal functions that we introduce now.

Let $L^{2}(X)=\left\{u: X \rightarrow \mathbb{C}: \int_{X}|u(x)|^{2} d x<\infty\right\}$ where $d x$ is the unique $G$ invariant Haar measure on $X$ such that $\int_{X} d x=1$. This vector space is given the standard $G$-action defined by $g \cdot u(x)=u\left(g^{-1}(x)\right)$ and is endowed with the canonical $G$-invariant hermitian product : $\left(u_{1}, u_{2}\right)=\int_{X} u_{1}(x) \overline{u_{2}(x)} d x$. The Peter-Weil theorem shows that $L^{2}(X)$ can be decomposed as a direct sum of $G$-irreducible subspaces $V_{i}$. The next step is to associate to each irreducible subspace $V_{i}$ a so-called zonal function $P_{V_{i}}$. A standard construction is the 
following: given an orthonormal basis $\left(u_{1}(x), \ldots, u_{d}(x)\right)$ of $V_{i}$, one can define

$$
\tilde{P}_{V_{i}}(x, y)=\frac{1}{d} \sum_{i=1}^{d_{i}} u_{i}(x) \overline{u_{i}(y)} .
$$

where $d_{i}=\operatorname{dim}\left(V_{i}\right)$. Since these functions are constant on $G$-orbits we can rewrite $\tilde{P}_{V_{i}}(x, y)=P_{V_{i}}(\tau(x, y))$. From this property comes the term zonal functions used to qualify them. From equation (11) it is easy to prove that these zonal functions verify the positivity property and do not depend of the chosen orthonormal basis. It turns out that, when the irreducible subspaces $V_{i}$ are pairwise non isomorphic, the cone of continuous positive $G$-invariant functions is exactly the set of linear combinations with non negative coefficients of the $P_{V_{i}}$ (see [5]). In the remaining of this paper we assume that this condition is satisfied. We moreover let $V_{0}$ denote the one-dimensional subspace associated to the trivial representation of $G$.

The so-called linear programming bounds are obtained with the following theorem :

Theorem 2.1 Let $P=\sum_{i} c_{i} P_{V_{i}}$ a linear combination of the zonal functions $P_{V_{i}}$ with a finite number of non zero coefficients. Assume furthermore that: $c_{i} \geq 0, c_{0}>0$ and $\Re(P)$ (the real part of $P$ ) is non-positive on $S$. Then any $S$-code verifies

$$
|\mathcal{V}| \leq \frac{P\left(\tau_{0}\right)}{c_{0}}
$$

where $\tau_{0}=\tau(x, x)$ for any $x$.

Proof : On one hand,

$$
\begin{aligned}
\sum_{x, y \in \mathcal{V}} P(\tau(x, y)) & =\sum_{x=y \in \mathcal{V}} P(\tau(x, y))+\sum_{x \neq y \in \mathcal{V}} P(\tau(x, y)) \\
& \leq|\mathcal{V}| P\left(\tau_{0}\right)
\end{aligned}
$$

On the other hand,

$$
\begin{aligned}
\sum_{x, y \in \mathcal{V}} P(\tau(x, y)) & =\sum_{x, y \in \mathcal{V}} c_{0} P_{0}(\tau(x, y))+\text { non negative terms } \\
& \geq|\mathcal{V}|^{2} c_{0} .
\end{aligned}
$$

\section{The case of unitary codes}

As a particular case we set $G=\mathbb{U}_{n}(\mathbb{C}) \times \mathbb{U}_{n}(\mathbb{C})$ and $X=\mathbb{U}_{n}(\mathbb{C})$. For $g=U \times V$ and $x=M$ we set $g x=U M V^{-1}$. In this context the orbit of a pair $(x, y)$ is 
characterized by the eigenvalues $\left(e^{i \theta_{1}}, \ldots, e^{i \theta_{n}}\right)$ of the unitary matrix $x y^{-1}$. The Peter-Weil theorem gives a decomposition of $L^{2}(X)$ into irreducible subspaces

$$
L^{2}(X)=\oplus\left(V_{\chi} \otimes V_{\bar{\chi}}\right)
$$

where the sum runs over all irreducible representations $V_{\chi}$ of $\mathbb{U}_{n}(\mathbb{C})$. It is worth noticing that the $G$-subspaces $V_{\chi} \otimes V_{\bar{\chi}}$ are pairwise non isomorphic. From this decomposition one can deduce the following theorem :

Proposition 3.1 The zonal functions associated to this decomposition are

$$
P_{\chi}(x, y)=\chi\left(x y^{-1}\right)
$$

where $\chi$ denotes any irreducible character of $\mathbb{U}_{n}(\mathbb{C})$.

The irreducible characters of $\mathbb{U}_{n}(\mathbb{C})$ are known to be finite dimensional and to have a nice description using Schur polynomials ([16]). We recall briefly some notations and definitions concerning those polynomials.

For any integer $k$, a partition of $k$ in $n$ parts is a finite decreasing sequence of $n$ non negative integers which sum exactly to $k$ ( $k$ is also called the degree of the partition). A partition $\lambda$ is dominating $\mu$ (noted $\lambda \succ \mu$ ) if $\forall r \leq n, \sum_{i=1}^{r} \lambda_{i} \geq$ $\sum_{i=1}^{r} \mu_{i}$. Given a partition $\lambda=\left[\lambda_{1}, \ldots, \lambda_{n}\right]$ we define the elementary symmetric polynomials $m_{\lambda} \in \mathbb{Z}\left[x_{1}, \ldots, x_{n}\right]$ as the renormalization to a monic polynomial of

$$
\sum_{\sigma \in \mathfrak{S}_{n}} x_{\sigma(1)}^{\lambda_{1}} x_{\sigma(2)}^{\lambda_{2}} \ldots x_{\sigma(n)}^{\lambda_{n}} .
$$

These polynomials form a basis of the set of symmetric polynomials $\mathbb{Z}\left[x_{1}, \ldots, x_{n}\right]^{\mathrm{Sym}}$. Schur polynomials have been intensively studied and have several definitions. For our purpose we will define the Schur polynomials $S_{\lambda}$ as

$$
S_{\lambda}=\sum_{\lambda \succ \mu} K_{\lambda, \mu} m_{\mu}
$$

where the $K_{\lambda, \mu} \in \mathbb{N}$ are the so called Kostka numbers. For more precision on those numbers see [15]. It is clear that Schur polynomials form another basis of $\mathbb{Z}\left[x_{1}, \ldots, x_{n}\right]^{\mathrm{Sym}}$.

It is well known that the irreducible polynomial characters of $\mathbb{U}_{n}(\mathbb{C})$ are expressed using Schur polynomials (we refer to [16] for details) in the following way: let $\left(e^{i \theta_{1}}, \ldots, e^{i \theta_{n}}\right)$ denote the eigenvalues of a unitary matrix $M$, and $\lambda$ a partition. Then

$$
\chi_{\lambda}(M)=S_{\lambda}\left(e^{i \theta_{1}}, \ldots, e^{i \theta_{n}}\right) .
$$

One obtains all irreducible characters of $\mathbb{U}_{n}(\mathbb{C})$ by multiplying the characters $\chi_{\lambda}$ by a relative power of $\operatorname{det}(M)=\prod e^{i \theta_{k}}$. All together, we obtain the theorem:

Theorem 3.2 For all partition $\lambda$ and $s \in \mathbb{Z}$, let

$$
P_{\lambda, s}\left(x_{1}, \ldots, x_{n}\right)=\left(x_{1} \ldots x_{n}\right)^{s} P_{\lambda}\left(x_{1}, \ldots, x_{n}\right) .
$$


These rational fractions give the zonal functions associated to the irreducible decomposition (3) in the following way: if $\chi \simeq \operatorname{det}^{s} \otimes \lambda$, if the eigenvalues of $x y^{-1}$ are $\left(e^{i \theta_{1}}, \ldots, e^{i \theta_{n}}\right)$, then

$$
P_{\chi}(x, y)=P_{\lambda, s}\left(e^{i \theta_{1}}, \ldots, e^{i \theta_{n}}\right) .
$$
Let

We are now almost ready to compute bounds for unitary codes $\mathcal{V} \subset \mathbb{U}_{n}(\mathbb{C})$.

$$
d_{\Sigma}^{2}\left(e^{i \theta_{1}}, \ldots, e^{i \theta_{n}}\right):=\frac{1}{2 n} \sum_{i=1}^{n}\left(1-\cos \theta_{i}\right)
$$

and

$$
d_{\Pi}^{2}\left(e^{i \theta_{1}}, \ldots, e^{i \theta_{n}}\right):=\frac{1}{2}\left(\prod_{i=1}^{n}\left(1-\cos \theta_{i}\right)\right)^{\frac{1}{n}} .
$$

and define $d_{\Sigma}^{2}(x, y)$ (resp. $\left.d_{\Pi}^{2}(x, y)\right)$ to be the above functions evaluated at the eigenvalues of $x y^{-1}$. These functions are related to the diversity sum and diversity product by

$$
\Sigma \mathcal{V}=\min _{\substack{x, y \in \mathcal{V} \\ x \neq y}} d_{\Sigma}(x, y) \text { and } \Pi \mathcal{V}=\min _{\substack{x, y \in \mathcal{V} \\ x \neq y}} d_{\Pi}(x, y) .
$$

We recall that the orbit of a pair $(x, y)$ is characterized by the eigenvalues of $x y^{-1}$, so $d_{\Sigma}$ and $d_{\Pi}$ are constant on $G$-orbits. We may now define, with the notations of Section II, the sets $S$ related to each diversity function:

$$
\begin{aligned}
S_{\Sigma}(\delta):=\left\{\left(e^{i \theta_{1}}, \ldots, e^{i \theta_{n}}\right): d_{\Sigma}\left(e^{i \theta_{1}}, \ldots, e^{i \theta_{n}}\right) \geq \delta\right\} \\
S_{\Pi}(\delta):=\left\{\left(e^{i \theta_{1}}, \ldots, e^{i \theta_{n}}\right): d_{\Pi}\left(e^{i \theta_{1}}, \ldots, e^{i \theta_{n}}\right) \geq \delta\right\} .
\end{aligned}
$$

\section{Analytic bounds of low degree}

From the explicit description of the zonal functions (4), we have deduced convenient polynomials of low degree which verify the positivity property. Using formula (2) we derive the analytic bounds of Theorems 4.1 and 4.2 .

Theorem 4.1 Let $\mathcal{V}$ be a unitary space time code with diversity sum $\Sigma \mathcal{V}$, the following upper bounds hold:

$$
\begin{gathered}
|\mathcal{V}| \leq \frac{2(\Sigma \mathcal{V})^{2}}{2(\Sigma \mathcal{V})^{2}-1}, \quad \text { if }(\Sigma \mathcal{V})^{2}>\frac{1}{2} \\
|\mathcal{V}| \leq \frac{8 n^{2}(\Sigma \mathcal{V})^{2}}{4 n^{2}(\Sigma \mathcal{V})^{2}-\left(2 n^{2}-1\right)}, \text { if }(\Sigma \mathcal{V})^{2}>\frac{2 n^{2}-1}{4 n^{2}}
\end{gathered}
$$




$$
\begin{gathered}
|\mathcal{V}| \leq \frac{16 n^{2}(\Sigma \mathcal{V})^{2}}{2 n(2 n-1)(\Sigma \mathcal{V})^{2}-\left(2 n^{2}-n-2\right)}, \\
\text { if }(\Sigma \mathcal{V})^{2} \geq \frac{2 n^{2}-n-2}{2 n(2 n-1)}
\end{gathered}
$$

Theorem 4.2 Let $\mathcal{V}$ be a unitary space time code with diversity product $\Pi \mathcal{V}$, the following upper bounds hold:

$$
\begin{gathered}
|\mathcal{V}| \leq \frac{2(\Pi \mathcal{V})^{2}}{2(\Pi \mathcal{V})^{2}-1}, \quad \text { if }(\Pi \mathcal{V})^{2}>\frac{1}{2} \\
|\mathcal{V}| \leq \frac{8 n(\Pi \mathcal{V})^{2}}{4 n(\Pi \mathcal{V})^{2}-(2 n-1)}, \text { if }(\Pi \mathcal{V})^{2}>\frac{2 n-1}{4 n}, n \geq 3 \\
|\mathcal{V}| \leq \frac{8(\Pi \mathcal{V})^{6}+4(\Pi \mathcal{V})^{4}+8(\Pi \mathcal{V})^{2}}{8(\Pi \mathcal{V})^{6}-\frac{1}{4}} \\
\text { if }(\Pi \mathcal{V})^{2} \geq \frac{1}{2}, n=2
\end{gathered}
$$

The proofs of these theorems are based on Theorem 2.1 and on the following lemma :

Lemma 4.3 Let $y_{j}=\cos \theta_{j}$ and $m_{\left[a_{1}, \ldots, a_{r}\right]}(y)$ the elementary symmetric polynomials in the $y_{j}=\cos \theta_{j}$. The following polynomials are linear combination of the zonal functions (4) with non negative coefficients:

$$
\begin{aligned}
& Q_{[0]}=1 \\
& Q_{[11]}=m_{[11]}(y)+\frac{(n-1)}{4} \\
& Q_{[1]}=m_{[1]}(y) \\
& Q_{[2]}=m_{[2]}(y)+m_{[11]}(y)-\frac{(n+1)}{4} \\
& Q_{[1,1,1]}=m_{[1,1,1]}(y)+\frac{(n-2)}{4} m_{[1]}(y) \\
& Q_{[2,1]}=m_{[2,1]}(y)+2 m_{[1,1,1]}(y)-\frac{1}{4} m_{[1]}(y) \\
& Q_{[3]}=m_{[3]}(y)+m_{[2,1]}(y)+m_{[1,1,1]}(y)-\frac{(n+2)}{4} m_{[1]}(y)
\end{aligned}
$$

Proof :[Theorem 4.1, Sketch] Let $s=\frac{1}{n} \sum_{j=1}^{n} \cos \theta_{j}$ so that $s=1-2 d_{\Sigma}^{2}$. We apply formula (2) with the polynomials : $P=Q_{[1]}-n s, P=\left(Q_{[1]}-n s\right)\left(Q_{[1]}+n\right)$, $P=\left(Q_{[1]}-n s\right) R$, where $R$ is the symmetrization of $\left(y_{1}+1\right)\left(y_{2}+1\right)$. 
Proof :[Theorem 4.2, Sketch] Let $p=2 d_{\pi}^{2}$. We apply formula (2) with the polynomials : $P=Q_{[1]}-n(p-1), P=Q_{[2]}+\frac{(n+1) p}{2} Q_{[1]}+\frac{(n+1)(2 n(p-1)+1)}{4}$ (if $n \geq 3$ ),

$P=Q_{[2]}+\left(\frac{p^{2}}{2}+2 p-1\right) Q_{[1]}+\left(p^{3}-\frac{1}{4}\right) \quad($ if $n=2)$.

\section{$5 \quad$ Numerical bounds}

Numerical programs give accurate approximations of the best linear programming bounds over a large interval of validity, not covered by the bounds proved in Section IV. The following curves plot the linear programming bound on the diversity functions as a function of the cardinality of the code. The programs optimize the choice of a polynomial in the variables $\left(\cos \theta_{1}, \ldots, \cos \theta_{n}\right)$, with degree at most equal to some parameter $D$. Increasing $D$ gives accurate results over a wider range of values for the diversity functions, but also increase the computational time. We use $D=19$ for $n=2$ and $D=13$ for $n=3$.

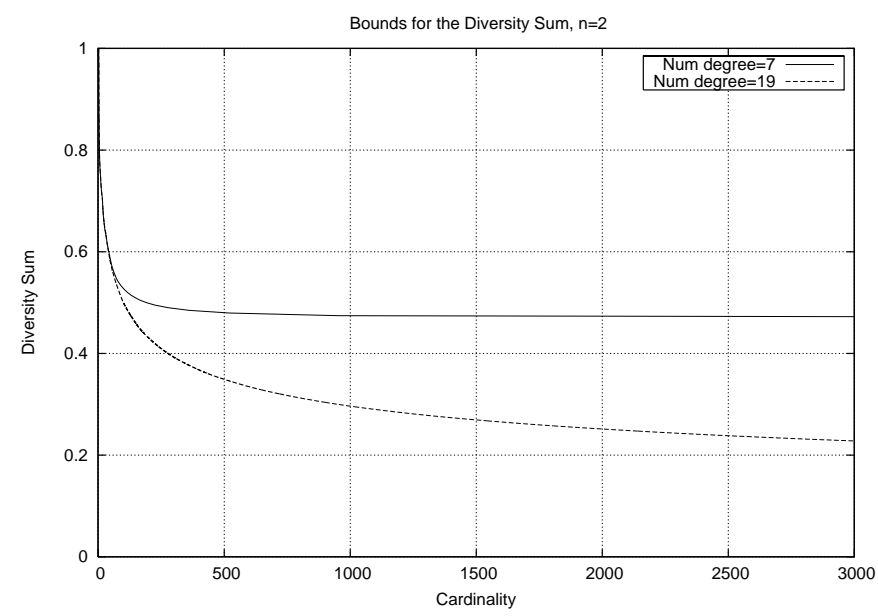



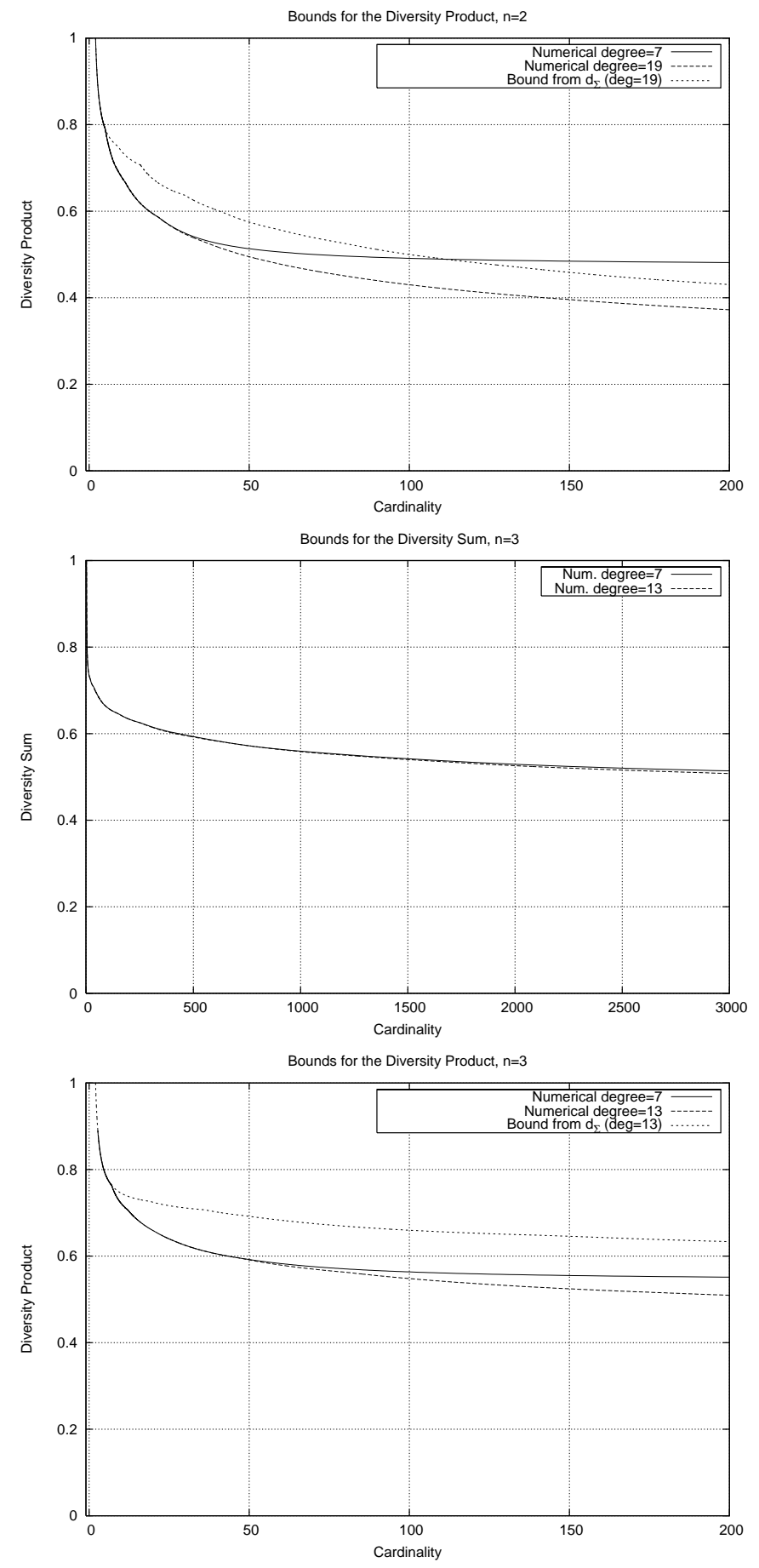


\begin{tabular}{|c|c|c|c|c|}
\hline $\mathrm{N}$ & 24 & 48 & 64 & 80 \\
\hline bounds in $[10]$ & 0.6746 & 0.6193 & 0.5969 & 0.5799 \\
\hline $\mathrm{B} 1[\underline{]}$ & 0.7598 & 0.6603 & 0.6131 & 0.5932 \\
\hline $\mathrm{B} 2[\underline{7}$ & 0.7794 & 0.6734 & 0.6235 & 0.6026 \\
\hline $\mathrm{LP} d_{\Sigma}$ & 0.6547 & 0.5797 & 0.5488 & 0.5254 \\
\hline $\mathrm{LP} d_{\Pi}$ & 0.5730 & 0.4989 & 0.4711 & 0.4504 \\
\hline
\end{tabular}

\begin{tabular}{|c|c|c|c|c|}
\hline $\mathrm{N}$ & 100 & 120 & 128 & 1000 \\
\hline bounds in [10] & 0.5632 & 0.5499 & 0.5452 & \\
\hline B1 [7] & 0.5578 & 0.5425 & 0.5347 & 0.3270 \\
\hline B2 [7] & 0.5654 & 0.5496 & 0.5415 & 0.3285 \\
\hline LP $d_{\Sigma}$ & 0.4999 & 0.4816 & 0.4753 & 0.2964 \\
\hline LP $d_{\Pi}$ & 0.4301 & 0.4144 & 0.4089 & 0.2574 \\
\hline
\end{tabular}

Table 1: $n=2$

\begin{tabular}{|c|c|c|c|c|}
\hline $\mathrm{N}$ & 24 & 48 & 64 & 80 \\
\hline $\mathrm{LP} d_{\Sigma}$ & 0.7178 & 0.6939 & 0.6797 & 0.6692 \\
\hline $\mathrm{LP} d_{\Pi}$ & 0.6431 & 0.5942 & 0.5752 & 0.5628 \\
\hline
\end{tabular}

\begin{tabular}{|c|c|c|c|c|}
\hline $\mathrm{N}$ & 100 & 120 & 128 & 1000 \\
\hline $\mathrm{LP} d_{\Sigma}$ & 0.6598 & 0.6532 & 0.6511 & 0.5586 \\
\hline $\mathrm{LP} d_{\Pi}$ & 0.5482 & 0.5369 & 0.5332 & 0.4330 \\
\hline
\end{tabular}

Table $2: n=3$

The Tables I and II compare the linear programming bounds for the dimensions 2 and 3 with the previous results of [10] and [7] and show an improvement in all cases.

These tables give upper bounds for the diversity when the cardinality $N$ is fixed. All entries except the ones of the last line (LP $\left.d_{\Pi}\right)$ are concerned with the diversity sum. The second line tabulates the bounds settled in [10, obtained using Coxeter upper bounds. The two bounds of [7] were obtained using sphere volume computations.

Moreover, concerning the diversity product, both our numerical results and analytic results (compare (6) and (9)) show a large gap between the bounds for diversity sum and diversity product, in favor of the diversity product. This is worth to point out since in previous publications bounds for the diversity product were essentially deduced from the trivial inequality $\Sigma \mathcal{V} \geq \Pi \mathcal{V}$ and hence appear to be weak.

\section{Conclusions}

In this paper we have developed the linear programming method for the unitary space time codes. We have obtained both numerical and analytic bounds. The 
results improve previously known bounds. Furthermore the linear programming method allows to deal with non-distance functions as the diversity product directly.

\section{Acknowledgment}

The authors would like to thank Christine Bachoc for her precious advises on the writing of this article.

\section{References}

[1] C. Bachoc, Linear programming bounds for codes in Grassmannian spaces, IEEE Trans. Inform. Theory, 52-5 (2006), pp. 2111-2125.

[2] A. Barg, P. Purkayastha, Bounds on ordered codes and orthogonal arrays, preprint, arxiv:cs.IT/0702033v1.

[3] J. Bierbrauer, A direct approach to linear programming bounds for codes and tms-nets, DCC, 42-2 (2007), pp. 127 - 143.

[4] P. Delsarte, V. I. Levenshtein,. Association schemes and coding theory IEEE Trans. Inform. Theory Vol. 44, n. 6, pp. 2477 - 2504, Oct. 1998.

[5] S. Bochner, Hilbert distances and positive definite functions, Ann. of Math. 42 (1941), pp. 647-656.

[6] B. M. Hochwald and T. L. Marzetta, Unitary space-time modulation for multiple antenna communication in Rayleigh flat fading. IEEE Trans. Inform. Theory, vol. 42 n. 2, pp. 543-564, 2000.

[7] G. Han, J. Rosenthal, Unitary Space-Time Constellation Analysis : An Upper Bound for the Diversity, IEEE Trans. Inform. Theory, vol. 52, n. 10, pp. 4713-4721, Oct. 2006.

[8] B. M. Hochwald, T. L. Marzetta, T. J. Richardson, W. Sweldens, R. Urbanke, Systematic design of unitary space-time constellations IEEE Trans. Inform. Theory Vol. 46, N. 6, pp. 1962-1973, Sep. 2000.

[9] G. A. Kabatiansky, V. I. Levenshtein, Bounds for packings on a sphere and in space, Probl. Inform.Transm. 14, pp. 1-17, 1978.

[10] X. B. Liang, X. G. Xia, Unitary signal constellations for differential spacetime modulation with two transmit antennas : Parametric codes, optimal designs and bounds, IEEE Trans. Inf. Theory, vol. 48, n. 8, pp. 2291-2322, Aug. 2002.

[11] A. Shokrollahi, Design of unitary space-time codes from representations of SU(2), Information Theory, 2001. Proceedings. 2001 IEEE International Symposium on Volume, Issue, 2001 Page(s):241 - 
[12] H. Tarnanen, Upper Bounds on Permutation Codes via Linear Programming, Eur. J. Comb. 20-1 (1999), pp. 101-114.

[13] L. Zheng and D. N. C. Tse, Communication on the Grassmann manifold: a geometric approach to the noncoherent multiple-antenna channel, vol. 48, n. 2, pp. 359383, Feb. 2002.

[14] J.H. Conway, N.J.A. Sloane, Sphere Packings, Lattices and Groups, Springer-Verlag, 1988.

[15] B. E. Sagan, The symmetric group, Graduate Texts in Mathematics, vol. 203, Second Ed. Springer-Verlag, 2001.

[16] W. Fulton Young Tableaux London Math. Society, Student Texts 35. 